EPJ Web of Conferences 19, 07008 (2012)

DOI: $10.1051 /$ epjconf/20121907008

(C) Owned by the authors, published by EDP Sciences, 2012

\title{
Searching for the Perseus arm in the anticenter direction
}

\author{
M. Monguió ${ }^{1, a}$, P. Grosbøl ${ }^{2}$ and F. Figueras ${ }^{1}$ \\ ${ }^{1}$ Dept. d'Astronomia i Meteorologia and IEEC-UB, ICC-Universitat de Barcelona \\ ${ }^{2}$ European Southern Observatory
}

\begin{abstract}
Here we propose a two step approach to provide new insights on the outer spiral arm pattern of the Milky Way: 1) a Strömgren photometric survey to map the stellar space density of moderate young type stars tracing the Perseus arm, and 2) a spectroscopic survey to determine the velocity perturbation due to the density wave through accurate Radial Velocity (RV, hereafter). First reduced preliminary data suggest an overdensity around $1.5-2 \mathrm{kpc}$, probably associated with the Perseus arm. Results from this project will present a significant step towards mapping the spiral structure between the second and the third galactic quadrant, where the determination of kinematic distances is not possible.
\end{abstract}

\section{MAPPING THE SPACE DENSITY USING UV BY $\beta$ PHOTOMETRY: PHOTOMETRIC SURVEY}

We have almost completed a $8 \square^{\circ}$ deep Strömgren uvbyH $\beta$ photometric survey for stars up to $u \sim 20$ with the Wide Field Camera (WFC) at the Isaac Newton Telescope (INT), La Palma. Target stars must be old enough to respond to the potential perturbation, but young enough to still have small intrinsic velocity dispersion. B5-A3 stars $(\sigma<15 \mathrm{~km} / \mathrm{s}$ according to [1]) are optimal for our studies. Strömgren photometry is the natural system to identify these stars and to compute accurate individual distances for them (10-15\% accuracy). Data from February 2009 run (3 $\left.\square^{\circ}\right)$ has been fully reduced and analyzed. Preliminary results (see Figure 1b) seems to indicate an stellar overdensity around 1.5-2 kpc. We estimate that this overdensity detection is in the range of 2-3 $\sigma$. Adding the double amount of stars obtained during January 2011 run we expect to be close to the five sigma detection. The spectroscopic targets are selected from our WFC/INT photometric survey of B5-A3 stars (300 stars/ $\left.\square^{\circ}\right)$.

Observational Program: Three successful observational runs have been developed (see Figure 1a) February 2009: $3 \square^{\circ}$ up to $u \sim 19$ already reduced and analyzed. 2) January 2011: 5 $\square^{\circ}$ up to $u \sim 20$ are being reduced. 3) February 2011: two bright nights, devoted to increase the sample for the first kiloparsec bins, where low statistics and saturation problems prevented us to reach the five sigma detection. The Strömgren $u$ filter defines the limiting magnitude of our sample. We checked that 2009 data allow us to ensure completeness only up to $2.2 \mathrm{kpc}(u \sim 19.5)$ for A3 stars. We did this comparison with a deeper region that was used as a standard field. During 2011 observations we increased the exposure times to ensure completeness up to $u=20$, so up to $2.5 \mathrm{kpc}$ for A3V stars.

Results: Distances, extinction and physical parameters have been obtained using the method from [2]. 1) From the distance histogram of B5-A3 stars we detect a possible overdensity around 1.5-2 kpc. 2) The extinction vs. distance plot shows a change in the slope. This tendency, if confirmed, will be consistent with a main dust lane on the inner side of the arm. In a standard density wave picture, this suggests that the co-rotation of the spiral pattern is outside this radius.

ae-mail: mmonguio@am.ub.es

This is an Open Access article distributed under the terms of the Creative Commons Attribution-Noncommercial License 3.0, which permits unrestricted use, distribution, and reproduction in any noncommercial medium, provided the original work is properly cited. 

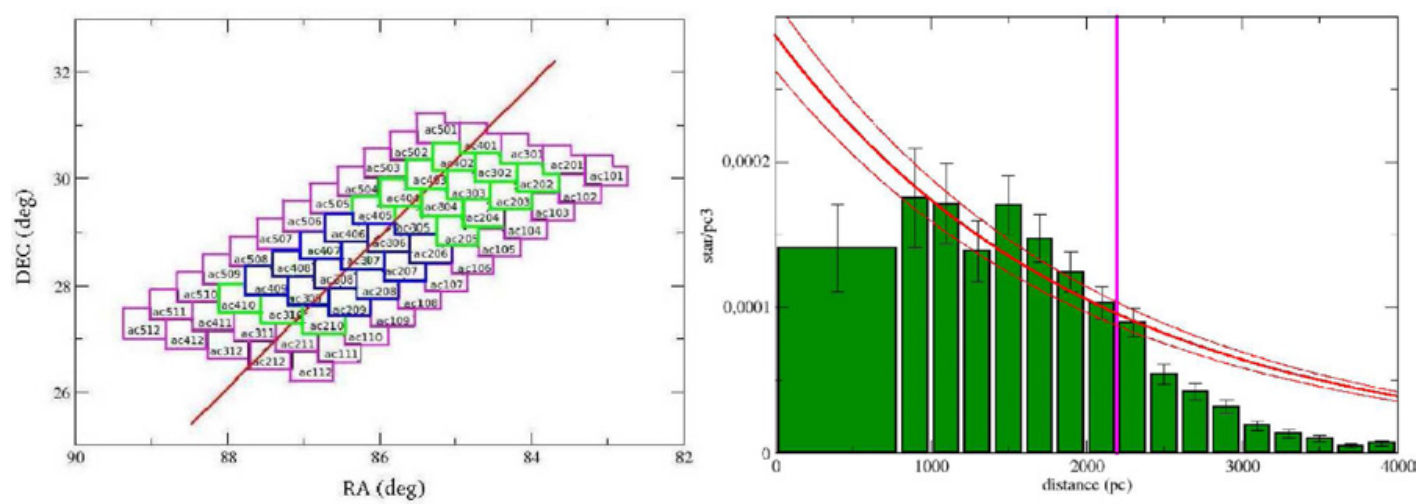

Figure 1. A) RA-DEC plot with the already observed WFC fields. In blue, data from 2009 (1), in green, data from January 2011 (2), in pink, data from February 2011 (3). Central point is at $(1, b)=(180.5,-0.25)$, following the warp and avoiding high extinction regions. Red line shows latitude $b=-0.25$. B) Stellar density distribution of B5A3 stars in the anticenter direction from $3 \square^{\circ}$ ( $~ 900$ stars). One sigma error bars are shown. Histogram complete up to $2.2 \mathrm{kpc}$, (pink vertical line). An exponential disk density law (red line) with scale length of $2 \mathrm{kpc}$ have been plotted for comparison. As a zero point of the exponential law we used $(2.87 \pm 0.24) 10^{-4} \mathrm{stars} / \mathrm{pc}^{3}$ at the Sun position, as obtained by [3] from Hipparcos data.

\section{VELOCITY PERTURBATION DUE TO THE SPIRAL PATTERN: SPECTROSCOPIC SURVEY}

Accurate RV for young B5-A3 stars are needed to trace the radial kinematic perturbation due to the Perseus arm in the anticenter direction. This direction allows us to minimize the influence of the galactic rotation. The RV perturbation due to the spiral pattern is rather uncertain and is expected to be small (amplitude $\mathrm{A}=2.5-5 \mathrm{~km} / \mathrm{s}$, e.g. [4]), so this fact imposes an important constrain on the accuracy required in our RV survey. Even if the real perturbation is smaller than expected, we will be able to set an upper limit. In order to develop that survey we are using AF2/WYFFOS at the William Herschel Telescope, La Palma. A high resolution grating has been selected to reach the $3-5 \mathrm{~km} / \mathrm{s}$ accuracy.

Obtained data: In November 2011 we observed spectra for $\sim 600$ stars (data is being reduced). A second epoch is needed to identify binary stars. Note that the kinematic sample do not need to be complete in distance, we just need to have enough stars in each bin to statistically detect the perturbation.

Simulations: We developed some simulations in order to establish the number of stars needed to achieve the detection of the perturbation and the RV accuracy required. The steps followed are: 1) use the distance distribution of the stars from the photometric sample (3 $\left.\square^{\circ}\right)$. 2) model the kinematic effect of the spiral arm with a sinusoidal as a function of distance: $R V=A(1-\cos (r / D s p))$, where: $A$ is the amplitude of the kinematic perturbation. $r$ is the heliocentric distance for each star obtained from the photometric sample. Dsp is the distance between the Sun and the expected locus of the Perseus arm ( $\sim 2 \mathrm{kpc}$, see above). The Sun is placed near the interarm region. 3) add an intrinsic RV dispersion as a function of the spectral type following [1]. 4) add an observational gaussian error in RV $\sigma=5 \mathrm{~km} / \mathrm{s}$ (upper limit). 5) add a 10\% error in distance. From several statistical tests we concluded that, to reach a $3 \sigma$ detection of a $2.5 \mathrm{~km} / \mathrm{s}$ amplitude perturbation, RV data for 800 stars is required. Taking into account that some of the observed stars will be binary (30\% according to [5]), we have increased the observing target list to 1000 stars. 
Assembling the Puzzle of the Milky Way

\section{References}

[1] Aumer, Binney, 2009, MNRAS 397,1286.

[2] Jordi, Masana, Figueras, Torra, 1997, A\&AS, 123, 83

[3] Murray, Penston, Binney, Houk, 1997, ESASP, 402, 485

[4] Fernández, Figueras, Torra, 2001, A\&A, 372, 833

[5] Evans, Bastian, Beletsky, et al., 2010, IAUS, 266, 35. 